\title{
Status and performance of the underground muon detector of the Pierre Auger Observatory
}

\section{Ana Martina Botti ${ }^{a, b, *}$ on behalf of the Pierre Auger ${ }^{c}$ Collaboration}

(a complete list of authors can be found at the end of the proceedings)

${ }^{a}$ Instituto de Tecnologías en Detección y Astropartículas (CNEA, CONICET, UNSAM), Buenos Aires, Argentina

${ }^{b}$ Department of Physics, FCEyN, University of Buenos Aires and IFIBA, CONICET, Buenos Aires, Argentina

${ }^{c}$ Observatorio Pierre Auger, Av. San Martín Norte 304, 5613 Malargüe, Argentina

E-mail: spokespersons@auger.org

The Auger Muons and Infill for the Ground Array (AMIGA) is an enhancement of the Pierre Auger Observatory, whose purpose is to lower the energy threshold of the observatory down to $10^{16.5} \mathrm{eV}$, and to measure the muonic content of air showers directly. These measurements will significantly contribute to the determination of primary particle masses in the range between the second knee and the ankle, to the study of hadronic interaction models with air showers, and, in turn, to the understanding of the muon puzzle. The underground muon detector of AMIGA is concomitant to two triangular grids of water-Cherenkov stations with spacings of 433 and $750 \mathrm{~m}$; each grid position is equipped with a $30 \mathrm{~m}^{2}$ plastic scintillator buried at $2.3 \mathrm{~m}$ depth. After the engineering array completion in early 2018 and general improvements to the design, the production phase commenced. In this work, we report on the status of the underground muon detector, the progress of its deployment, and the performance achieved after two years of operation. The detector construction is foreseen to finish by mid-2022.

$37^{\text {th }}$ International Cosmic Ray Conference (ICRC 2021)

July 12 th $-23 r d, 2021$

Online - Berlin, Germany

\footnotetext{
${ }^{*}$ Presenter
} 

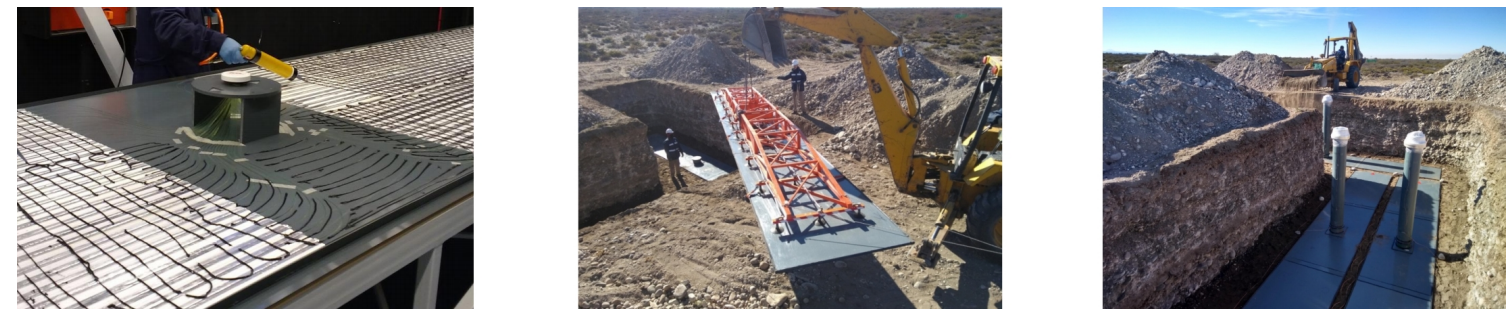

Figure 1: (Left) scintillator module under construction. (Middle) module being deployed at the field. (Right) a full UMD position deployed, before being buried.

\section{Introduction}

The first surface detector of the Pierre Auger Observatory [1] consisted of an array of about 1600 water-Cherenkov stations distributed in a triangular grid with $1500 \mathrm{~m}$ spacing (SD-1500) covering a total area of $3000 \mathrm{~km}^{2}$. It was meant to detect cosmic rays with full efficiency at energies above $10^{18.5} \mathrm{eV}$. After its completion, in 2008, two denser arrays with reduced spacings of $750 \mathrm{~m}$ (SD-750) and $433 \mathrm{~m}$ (SD-433) were deployed as a part of the Auger Muon and Infill for the Ground Array (AMIGA) [2] enhancement. The energy threshold of the surface detector was then extended to $10^{16.5} \mathrm{eV}$. In addition, AMIGA is also aimed at obtaining direct measurements of the muonic component of air showers with an underground muon detector (UMD).

The UMD consists of an array of scintillators associated with the water-Cherenkov detectors of the SD-750 and SD-433. At each grid position, three $10 \mathrm{~m}^{2}$ modules are buried at a depth of $2.3 \mathrm{~m}$ to shield them from the electromagnetic component of air showers [3]. Each module consists, in turn, of 64 scintillator strips with wavelength-shifting optical fiber coupled to an array of silicon photomultipliers (SiPMs) [4]. Pictures of a module commissioning are presented in Fig. 1: we show a module under construction (left) and deployment (middle), along with an image of a complete deployed station (right) just before being overburden with soil. The whole UMD array will have 73 positions, 219 modules, and over 14000 SiPMs. The deployment status, at the time of writing, is presented in Fig. 2 where we discriminate the positions shared with the SD-1500 (blue circles) and only AMIGA (gray circles for the SD-750 and red for the SD-433) grids. The big hexagon (dashed-gray line) denotes the limits of the whole UMD array, while the small hexagon (dashed-red line) indicates the location of the SD-433 array. The deployment and commissioning of the UMD are currently undergoing at a pace of 2 full-positions $\left(6 \times 10 \mathrm{~m}^{2}\right)$ per month. The full circles in Fig. 2 indicate the positions where the UMD scintillators are already buried. A total of 24 stations (about $35 \%$ of the whole array) are deployed: 20 stations of the SD-750 and 4 of the SD-433.

In addition to the UMD, both scintillator [5] and radio detectors [6] will be deployed on top of the water-Cherenkov detectors in the SD-1500 as part of the Pierre Auger Observatory upgrade [7]. This will improve the separation of the muonic and electromagnetic components in air showers and, in turn, the cosmic-ray mass identification at the highest energies.

\section{The underground muon detector}

Each acquisition channel of the UMD consists of a scintillator strip with optical fiber coupled to a SiPM. The buried modules are segmented in 64 of these channels, whose outputs are processed 


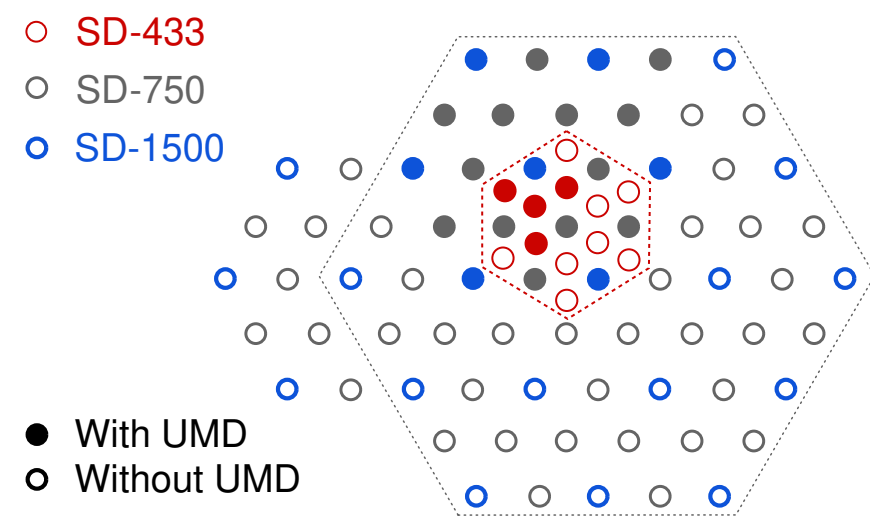

Figure 2: Schematic of the AMIGA array. The big hexagon denotes the limits of the UMD array, while the small hexagon indicates the limits of the SD-433 array.

in two different ways: (i) in 64 binary traces, the so-called binary acquisition mode, and (ii) in two waveforms, dubbed ADC mode. By implementing both acquisition modes, we attain a dynamic range that extends from a single muon up to a few tens of muons per $\mathrm{m}^{2}$.

Within the binary channel [4], each SiPM signal is processed independently through a preamplifier, a fast-shaper, and a discriminator integrated into two 32-channel application-specific integrated circuits. Then, the discriminator outputs are sampled with a field-programmable gate array in 64 binary traces with a sampling speed of $320 \mathrm{MHz}(3.125 \mathrm{~ns})$ and a total event time of $6.4 \mu \mathrm{s}$. For each channel and time sample, the output consists of a " 0 " (" 1 ") if the fast-shaper signal is below (above) the discriminator threshold. This threshold is set at 2.5 photo-equivalents (PE) to filter most of the SiPM dark current. The number of impinging particles is finally inferred by searching sequences of " 1 "s in the binary traces.

In the ADC acquisition mode [8], the 64 SiPM signals are summed with two steps of adders and then processed with low- and high-gain amplifiers. The outputs are sampled with analog-todigital converters into two waveforms with a sampling speed of $160 \mathrm{MHz}(6.25 \mathrm{~ns})$. By computing the signal charge and dividing it by the mean of a single muon, we can determine the number of impinging particles with this mode.

The binary acquisition is hardware-limited by the segmentation of the modules: if more than 63 segments are activated simultaneously, then the binary mode is saturated. This mode is designed to measure low densities with minimized uncertainties as fluctuations of the energy deposit in the scintillator, and subsequent photo-production have a reduced impact on the resulting sequence of "1"s. However, uncertainties associated with the binary mode grow with the number of muons as the impinging particles pile up. Not only does the ADC mode extend the saturation point of the UMD but also, it allows for improved measurements in a region where, despite not being saturated, the binary mode introduces large fluctuations.

The trigger system of the UMD depends on the SD-750/433 triggers both at the local station and global array level [9]. Once a local trigger condition is satisfied in a water-Cherenkov detector, a signal is sent to the UMD modules, and data is temporarily stored in internal memory. When an array trigger condition is met, the data is sent to the central acquisition system for permanent 


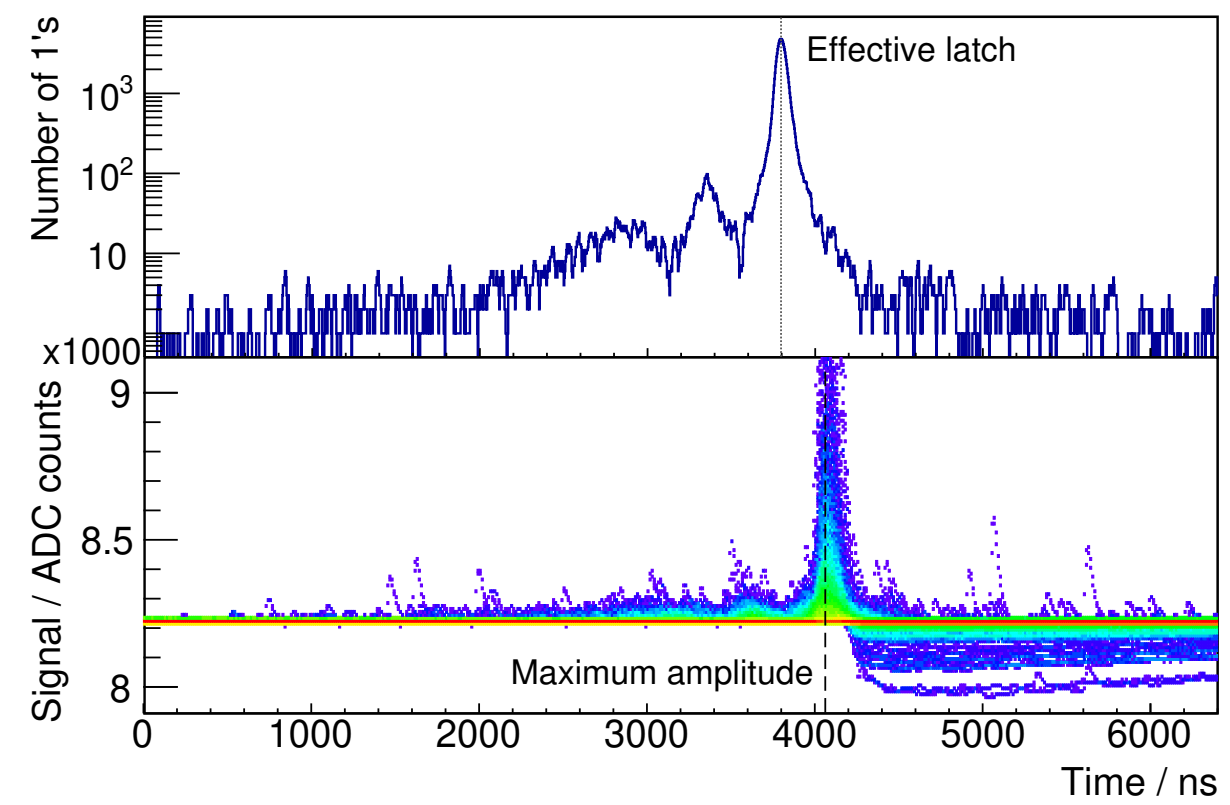

Figure 3: (Top) binary channel raw traces. Signal accumulation around the trigger scope can be seen. Randomly distributed entries produced by background noise are also observed. (Bottom) raw traces of the ADC low-gain channel. Similarly to the binary channel, accumulation of signal can be seen within the shower scope, along with uniformly distributed noise.

storage. An example of UMD traces obtained during two months of air shower data in a single UMD module is displayed in Fig. 3. We present the addition of all binary traces in the top panel, and the overlap of all ADC traces at the bottom. The three peaks between 2500 and $4200 \mathrm{~ns}$ correspond to the different local trigger types of the water-Cherenkov detector [9]. Entries beyond this range are produced mainly by the noise of both the SiPM and the scintillator/optical fiber system. We denominate the time bin of maximum accumulation in the binary channel as the effective latch. This bin is of particular importance for the calibration of the ADC mode. It is worth noting the delay of about 150 ns between binary and ADC channel mainly produced by the different electronic responses, and undershoot in the ADC originated by signals with a high amplitude.

\section{Calibration}

To determine the muonic content in air showers with the UMD it is necessary to interpret the previously displayed traces in terms of impinging particles. For the binary channel, this is defining a strategy to translate the sequences of "1"s into a number of particles, i.e., finding the time width for which the amplitude of a typical muon pulse is above the discriminator threshold. In the ADC mode, we map the traces to particles dividing the total signal charge by the mean of a single muon. During the calibration process, both the discriminator threshold level and the single-muon charge are determined [10].

The first step of the procedure is to set the operating point of the SiPMs using their thermal noise: the dark-count rate is measured as a function of an amplitude threshold using different reverse-bias voltages, $V_{\text {bias. }}$. In the left panel of Fig. 4 , we show the rate of dark-counts as a function 

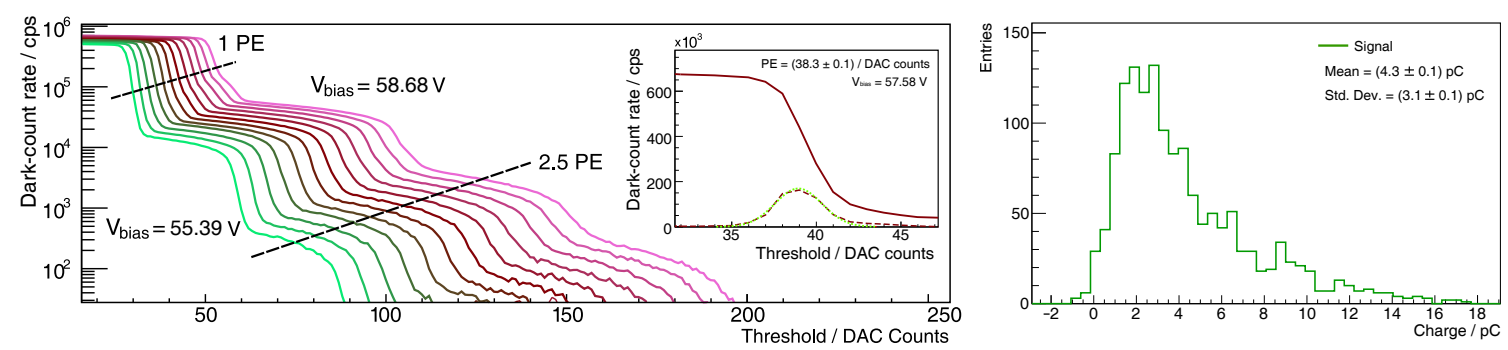

Figure 4: (Left) Dark-counts per second (cps) as a function of the discriminator threshold for different $V_{\text {bias }}$ with an individual SiPM. In the inset, we show one rate curve (solid brown) and its derivative (dashed brown) fitted with a Gaussian distribution (dotted green) to obtain the corresponding PE amplitude. (Right) Example of single-muon charge histogram. The calibration histogram is filled by selecting background muon signals with the binary channel.

of an amplitude threshold for different values of $V_{\text {bias }}$; the mean amplitude of 1 and 2.5 PE signals are indicated for illustration. It is apparent how the dark-count rate and PE amplitude shift towards higher values when increasing the $V_{\text {bias. }}$. In the inset, we present the determination of the PE amplitude for one of the rate curves: the PE amplitude is located between the transition of the first two consecutive plateaus (solid-brown line) and is fitted (dotted-green line) after differentiating the rate curve (dashed-brown line). By repeating this procedure for all curves, we determine the $1 \mathrm{PE}$ mean amplitude as a function of the $V_{\text {bias. }}$. This amplitude is proportional to the SiPM gain and becomes 0 when $V_{\text {bias }}$ is equal to the minimum voltage needed to operate the SiPMs in Geiger mode, named, breakdown voltage or $V_{\mathrm{br}}$. Once the $V_{\mathrm{br}}$ is determined, we can set $V_{\mathrm{bias}}=V_{\mathrm{br}}+3.5 \mathrm{~V}$, and the discriminator threshold of the binary mode at 2.5 PE. This routine, replicated in all the UMD channels, allows us to uniformize the module response by setting the same over-voltage of $3.5 \mathrm{~V}$ to all SiPMs.

To obtain the mean charge of single muons in the ADC channel, an algorithm runs parallel to the shower acquisition: when a UMD module receives a local trigger signal from the water-Cherenkov detector, the algorithm looks for an exclusive single-muon signature in the 64 binary traces, which consists of a sequence between four and twelve "1"s [10]. If this condition is fulfilled, the signal in the ADC channel corresponds to a single muon, and we compute the charge by integrating the trace in a $200 \mathrm{~ns}$ window. We present an example of the resulting charge histogram for the ADC acquisition mode in the right panel of Fig. 4 where we obtained a mean charge of $(4.3 \pm 0.1) \mathrm{pC}$ and a standard deviation of $(3.1 \pm 0.1) \mathrm{pC}$. We can fill a calibration histogram with data close in time to an air shower to reduced uncertainties in the determination of the number of impinging particles or periodically to assess the UMD performance (see Section 4).

\section{Long-term stability and array uniformity}

The UMD modules will be operated at the Pierre Auger site, where environmental conditions cannot be controlled, for several years. Therefore, monitoring the detector performance is of utmost importance to guarantee the well-functioning of the array.

The algorithm to extract calibration data is integrated into the UMD electronics firmware and requests two parameters: the effective latch bin of the binary channel and the delay between binary 

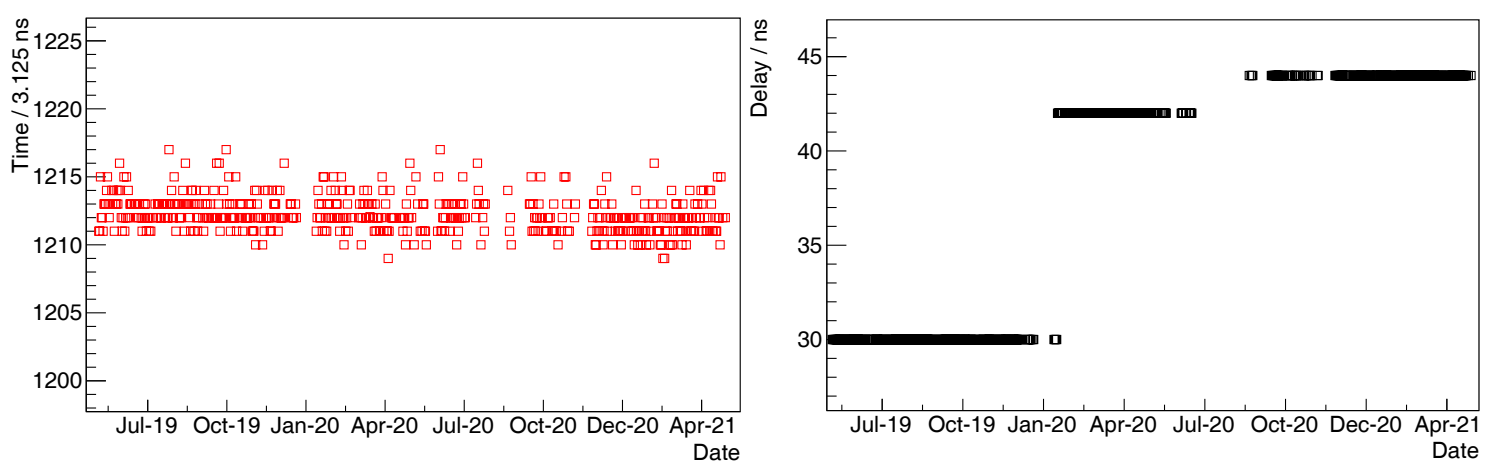

Figure 5: (Left) mean start time and effective latch in the binary channel as a function of time. (Right) mean delay between the start time of the binary channel and the maximum amplitude of the ADC trace. Gaps match the periods where the module was out of acquisition or under maintenance.

and ADC as already indicated in Fig. 3. We need the latch to look for the exclusive single-muon signature in the binary trace: the sequence of more than four and less than twelve " 1 "s is search in a $62.5 \mathrm{~ns}$ window around this bin. The delay is used to define the start time of the $200 \mathrm{~ns}$ window to integrate the charge of the ADC signal.

The stability of these two parameters is monitored by inspecting the full binary and ADC traces, only available in data permanently stored after global array triggers. As an example, we show in Fig. 5 the effective latch (left) and the delay (right) in an individual module as a function of time for about two years of operation. Jumps in the latter were produced when the UMD firmware was updated to include new functionalities. Still, the delay remains stable between firmware updates, and the jumps only denote the importance of updating this parameter when modifying the electronics firmware. The gaps in the timeline correspond to periods where the module was out of acquisition and/or under maintenance.

The SiPMs are operated with a temperature compensation mechanism that stabilizes the gain among the whole UMD array to assure the same detection efficiency through the years. However, the dependency of the dark-count rate on the temperature cannot be controlled. As an example, we present in Fig. 6 the electronics temperature (red squares and right y-axis) and the rate of dark-counts with an amplitude above 2.5 PE (black circles and left y-axis) as a function of time for an example module. The dark-count rate is obtained after averaging the $64 \mathrm{SiPMs}$; we present both temperature and rate as the mean per hour. It is apparent how the SiPM noise is modulated by the temperature: the mean rate per SiPM varies between 250 and $500 \mathrm{~Hz}$, a factor of two between summer and winter. For the 64 SiPMs, this implies a probability of having a "1" produced by noise between $10 \%$ and $20 \%$ in a time window of $6.4 \mu \mathrm{s}$.

After deploying a module in the field, we need to set up the algorithm to extract data for the ADC calibration. After configuring the latch and delay, the output of the calibration algorithm is stored in the acquisition server and can be subsequently used offline to extract the single-muon charge. As we extract this data in real-time, it is convenient for module monitoring and sanity-check purposes.

To illustrate the performance of the calibration, we present in the left panel of Fig. 7, the number of events matching the muon selection criteria per hour as a function of time for one 


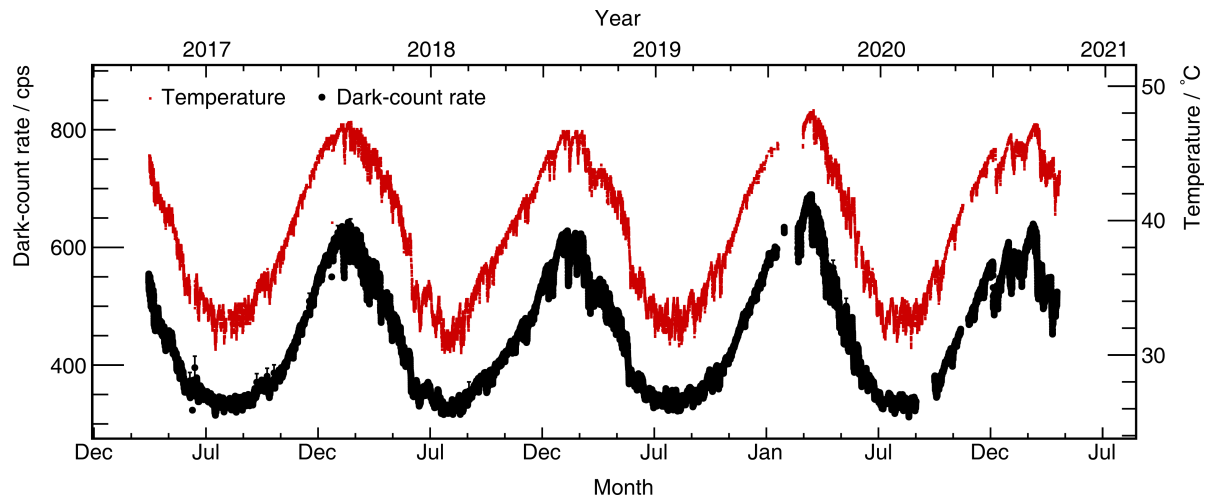

Figure 6: Temperature (right y-axis) and mean dark-count rate (left y-axis) per SiPM as a function of time, averaged every one hour.
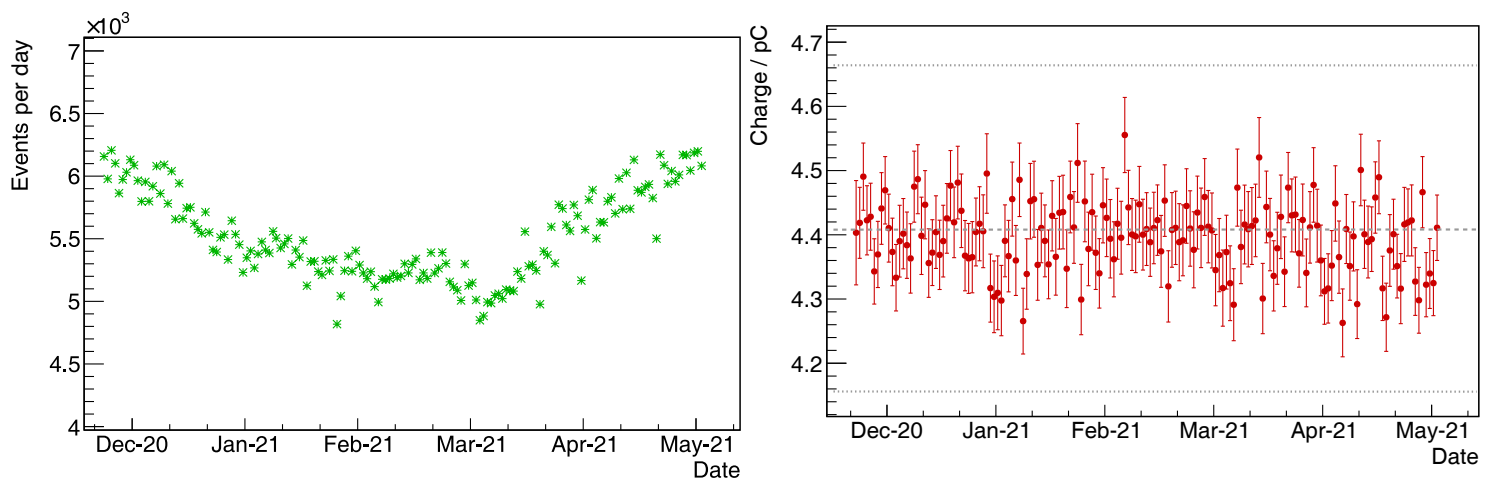

Figure 7: Number of events (left) and mean charge of the muon signal with error bars indicating one standard deviation (right) as a function of time. Each data point is extracted from histograms filled with 24 hours of calibration data.

example module; a seasonal modulation seems to appear with an amplitude of about $10 \%$ from the mean. We also present the charge of the single-muon signal every 24 hours in the right panel of Fig. 7, determined from calibration histograms as in Fig. 4. The mean value obtained for the whole period (dashed-gray line) is $(4.408 \pm 0.004) \mathrm{pC}$ with a standard deviation of $(0.059 \pm 0.003) \mathrm{pC}$, which corresponds to a mean fluctuation of $(\sim 1.3 \%)$ in the whole analyzed period. To better illustrate this, the $5 \%$ fluctuation of the mean, corresponding to about four standard deviations, is shown in dotted-gray lines. This indicates that the calibration method is robust and stable, even under temperature variations.

We also assessed the uniformity of the array using data from 21 modules so far deployed in the field. For each one, we obtained the mean charge averaged over six months; results are presented in the left panel of Fig. 8. The mean obtained over the array is $(5.69 \pm 0.08) \mathrm{pC}$ with a standard deviation of $(0.68 \pm 0.06) \mathrm{pC}$. Finally, we computed the mean dark-count rate for all modules in the array, and we present the result in the right panel of Fig. 8. We estimated the rate averaging data from one month in summer (February 2021) and one in winter (August 2020). Histograms are normalized since the total number of modules increased between the two time periods. The seasonal dependence of the SiPM noise is apparent. 

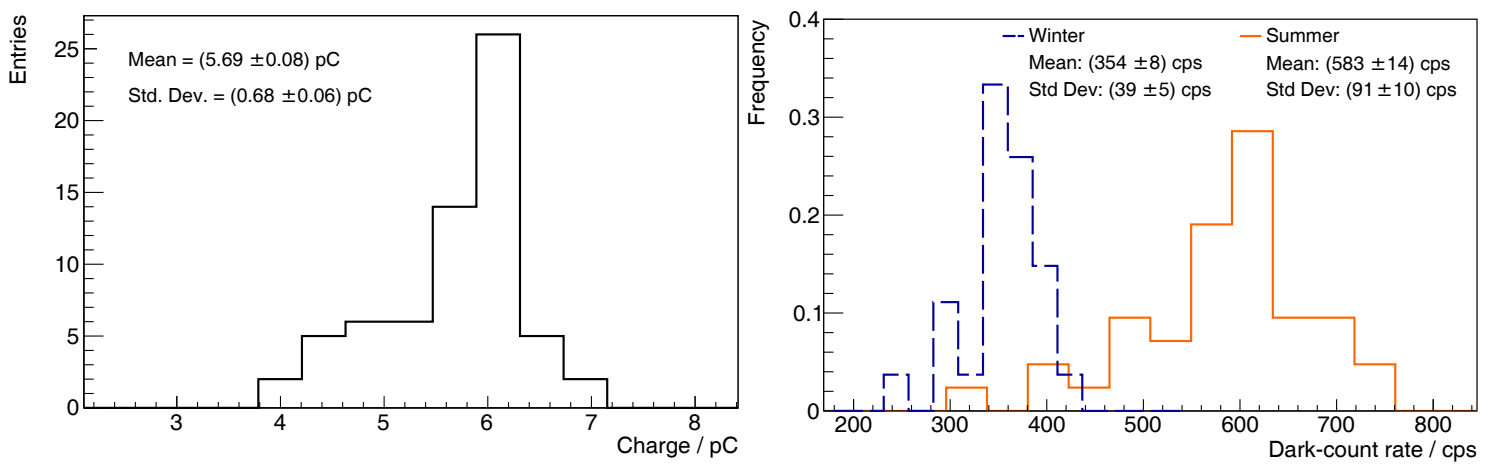

Figure 8: (Left) Mean charge per module in the ADC channel. Each entry corresponds to the mean charge of a UMD module averaged in a period of one month. (Right) Mean rate of dark-counts with amplitude above 2.5 PE per module. Results are shown after averaging one month in summer and one in winter. Histograms are normalized to the total number of modules in acquisition during the time period.

\section{Summary and outlook}

The status and performance of the AMIGA underground muon detector were presented. The deployment of the UMD is undergoing, having 24 stations out of 73 already deployed (35\% of the whole array), and is foreseen to finish by mid-2022.

We discussed the performance of the UMD calibration data and parameters for the two acquisition modes: binary and ADC showing that the calibration method is robust to work with the 219 modules and more than $14000 \mathrm{SiPMs}$ in the whole array that will be acquiring data for several years.

Quality air shower data is already available and will significantly increase with the deployment of new positions. High-level physics analyses with UMD data will soon commence.

\section{References}

[1] A. Aab et al. [Pierre Auger Coll.], Nucl. Instrum. Meth. A 798 (2015) 172-213, [1502.01323].

[2] A. Etchegoyen et al. [for the Pierre Auger Coll.], Proc. 30th Int. Cosmic Ray Conf., Mérida, Mexico (2007), 1191-1194, [0710.1646].

[3] A. Aab et al. [Pierre Auger Coll.], J. Instrum. 11 (2016) P02012, [1605.01625].

[4] A. Aab et al. [Pierre Auger Coll.], J. Instrum. 12 (2017) P03002, [1703.06193].

[5] G. Cataldi et al. [for the Pierre Auger Coll.], these proceedings.

[6] F. Schlüter et al. [for the Pierre Auger Coll.], these proceedings.

[7] A. Aab et al. [Pierre Auger Coll.], (2016) [1604.03637].

[8] A. Aab et al. [Pierre Auger Coll.], J. Instrum. 11 (2020) P01026, [2011.06633].

[9] A. Aab et al. [Pierre Auger Coll.], Nucl. Instrum. Meth. A 613 (2010) 29-39, [1111. 6764].

[10] A. Aab et al. [Pierre Auger Coll.], J. Instrum. 04 (2021) P04003, [2012 . 08016]. 


\section{The Pierre Auger Collaboration}

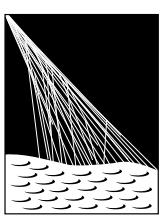

PIERRE

AUSGERVATORY

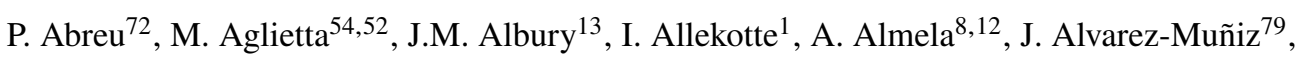
R. Alves Batista ${ }^{80}$, G.A. Anastasi ${ }^{63,52}$, L. Anchordoqui ${ }^{87}$, B. Andrada ${ }^{8}$, S. Andringa ${ }^{72}$, C. $\mathrm{Aramo}^{50}$, P.R. Araújo Ferreira ${ }^{42}$, J. C. Arteaga Velázquez ${ }^{67}$, H. Asorey $^{8}$, P. Assis ${ }^{72}$, G. Avila ${ }^{11}$, A.M. Badescu ${ }^{75}$, A. Bakalova ${ }^{32}$, A. Balaceanu ${ }^{73}$, F. Barbato ${ }^{45,46}$, R.J. Barreira Luz $^{72}$, K.H. Becker ${ }^{38}$, J.A. Bellido ${ }^{13,69}$, C. Berat ${ }^{36}$, M.E. Bertaina ${ }^{63,52}$, X. Bertou ${ }^{1}$, P.L. Biermann ${ }^{b}$, V. Binet ${ }^{6}$, K. Bismark ${ }^{39,8}$, T. Bister ${ }^{42}$, J. Biteau ${ }^{37}$, J. Blazek ${ }^{32}$, C. Bleve ${ }^{36}$, M. Boháčová ${ }^{32}$, D. Boncioli ${ }^{57,46}$, C. Bonifazi ${ }^{9,26}$, L. Bonneau Arbeletche ${ }^{21}$, N. Borodai ${ }^{70}$, A.M. Botti ${ }^{8}$, J. Brack ${ }^{d}$, T. Bretz ${ }^{42}$, P.G. Brichetto Orchera ${ }^{8}$, F.L. Briechle ${ }^{42}$, P. Buchholz ${ }^{44}$, A. Bueno ${ }^{78}$, S. Buitink ${ }^{15}$, M. Buscemi ${ }^{47}$, M. Büsken ${ }^{39,8}$, K.S. Caballero-Mora ${ }^{66}$, L. Caccianiga ${ }^{59,49}$, F. Canfora ${ }^{80,81}$, I. Caracas ${ }^{38}$, J.M. Carceller ${ }^{78}$, R. Caruso ${ }^{58,47}$, A. Castellina ${ }^{54,52}$, F. Catalani ${ }^{19}$, G. Cataldi ${ }^{48}$, L. Cazon ${ }^{72}$, M. Cerda ${ }^{10}$, J.A. Chinellato ${ }^{22}$, J. Chudoba ${ }^{32}$, L. Chytka ${ }^{33}$, R.W. Clay ${ }^{13}$, A.C. Cobos Ceruttii ${ }^{7}$, R. Colalillo ${ }^{60,50}$, A. Coleman ${ }^{93}$, M.R. Coluccia ${ }^{48}$, R. Conceição ${ }^{72}$, A. Condorelli ${ }^{45,46}$, G. Consolati ${ }^{49,55}$, F. Contreras ${ }^{11}$, F. Convenga ${ }^{56,48}$, D. Correia dos Santos $^{28}$, C.E. Covault ${ }^{85}$, S. Dasso ${ }^{5,3}$, K. Daumiller ${ }^{41}$, B.R. Dawson ${ }^{13}$, J.A. Day ${ }^{13}$, R.M. de Almeida $^{28}$, J. de Jesús ${ }^{8,41}$, S.J. de Jong ${ }^{80,81}$, G. De Mauro ${ }^{80,81}$, J.R.T. de Mello Neto ${ }^{26,27}$, I. De Mitri ${ }^{45,46}$, J. de Oliveira ${ }^{18}$, D. de Oliveira Franco ${ }^{22}$, F. de Palma ${ }^{56,48}$, V. de Souza $^{20}$, E. De Vito ${ }^{56,48}$, M. del Río ${ }^{11}$, O. Deligny ${ }^{34}$, L. Deval ${ }^{41,8}$, A. di Matteo $^{52}$, C. Dobrigkeit ${ }^{22}$, J.C. D’Olivo ${ }^{68}$, L.M. Domingues Mendes ${ }^{72}$, R.C. dos Anjos ${ }^{25}$, D. dos Santos $^{28}$, M.T. Dova ${ }^{4}$, J. Ebr ${ }^{32}$, R. Engel ${ }^{39,41}$, I. Epicoco ${ }^{56,48}$, M. Erdmann ${ }^{42}$, C.O. Escobar ${ }^{a}$, A. Etchegoyen ${ }^{8,12}$, H. Falcke ${ }^{80,82,81}$, J. Farmer ${ }^{92}$, G. Farrar ${ }^{90}$, A.C. Fauth ${ }^{22}$, N. Fazzini ${ }^{a}$, F. Feldbusch ${ }^{40}$, F. Fenu ${ }^{54,52}$,

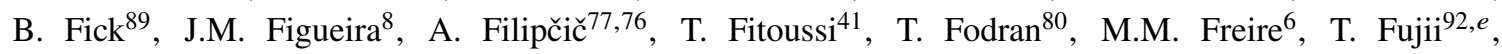
A. Fuster $^{8,12}$, C. Galea ${ }^{80}$, C. Galelli ${ }^{59,49}$, B. García ${ }^{7}$, A.L. Garcia Vegas ${ }^{42}$, H. Gemmeke ${ }^{40}$, F. Gesualdi ${ }^{8,41}$, A. Gherghel-Lascu ${ }^{73}$, P.L. Ghia ${ }^{34}$, U. Giaccari ${ }^{80}$, M. Giammarchi ${ }^{49}$, J. Glombitza ${ }^{42}$, F. Gobbi ${ }^{10}$, F. Gollan ${ }^{8}$, G. Golup ${ }^{1}$, M. Gómez Berisso ${ }^{1}$, P.F. Gómez Vitale ${ }^{11}$, J.P. Gongora ${ }^{11}$, J.M. González ${ }^{1}$, N. González ${ }^{14}$, I. Goos ${ }^{1,41}$, D. Góra ${ }^{70}$, A. Gorgi ${ }^{54,52}$, M. Gottowik ${ }^{38}$, T.D. Grubb ${ }^{13}$, F. Guarino ${ }^{60,50}$, G.P. Guedes ${ }^{23}$, E. Guido ${ }^{52,63}$, S. $\mathrm{Hahn}^{41,8}$, P. $\mathrm{Hamal}^{32}$, M.R. Hampel ${ }^{8}$, P. Hansen ${ }^{4}$, D. Harari ${ }^{1}$, V.M. Harvey ${ }^{13}$, A. Haungs ${ }^{41}$, T. Hebbeker ${ }^{42}$, D. Heck ${ }^{41}$, G.C. Hill ${ }^{13}$, C. Hojvat ${ }^{a}$, J.R. Hörandel ${ }^{80,81}$, P. Horvath ${ }^{33}$, M. Hrabovský ${ }^{33}$, T. Huege ${ }^{41,15}$, A. Insolia ${ }^{58,47}$, P.G. Isar $^{74}$, P. Janecek ${ }^{32}$, J.A. Johnsen ${ }^{86}$, J. Jurysek ${ }^{32}$, A. Kääpä ${ }^{38}$, K.H. Kampert ${ }^{38}$, N. Karastathis ${ }^{41}$, B. Keilhauer ${ }^{41}$, J. Kemp ${ }^{42}$, A. Khakurdikar ${ }^{80}$, V.V. Kizakke Covilakam $^{8,41}$, H.O. Klages ${ }^{41}$, M. Kleifges ${ }^{40}$, J. Kleinfeller ${ }^{10}$, M. Köpke ${ }^{39}$, N. Kunka ${ }^{40}$, B.L. Lago ${ }^{17}$, R.G. Lang ${ }^{20}$, N. Langner ${ }^{42}$, M.A. Leigui de Oliveira ${ }^{24}$, V. Lenok $^{41}$, A. Letessier-Selvon ${ }^{35}$, I. LhenryYvon $^{34}$, D. Lo Presti ${ }^{58,47}$, L. Lopes ${ }^{72}$, R. López ${ }^{64}$, L. Lu ${ }^{94}$, Q. Luce ${ }^{39}$, J.P. Lundquist ${ }^{76}$, A. Machado Payeras $^{22}$, G. Mancarella ${ }^{56,48}$, D. Mandat ${ }^{32}$, B.C. Manning ${ }^{13}$, J. Manshanden ${ }^{43}$, P. Mantsch ${ }^{a}$, S. Marafico ${ }^{34}$, A.G. Mariazzi ${ }^{4}$, I.C. Mariş ${ }^{14}$, G. Marsella ${ }^{61,47}$, D. Martello ${ }^{56,48}$, S. Martinelli ${ }^{41,8}$, O. Martínez Bravo ${ }^{64}$, M. Mastrodicasa ${ }^{57,46}$, H.J. Mathes ${ }^{41}$, J. Matthews ${ }^{88}$, G. Matthiae ${ }^{62,51}$, E. Mayotte ${ }^{38}$, P.O. Mazur ${ }^{a}$, G. MedinaTanco $^{68}$, D. Melo $^{8}$, A. Menshikov ${ }^{40}$, K.-D. Merenda ${ }^{86}$, S. Michal ${ }^{33}$, M.I. Micheletti ${ }^{6}$, L. Miramonti ${ }^{59,49}$, S. Mollerach ${ }^{1}$, F. Montanet ${ }^{36}$, C. Morello ${ }^{54,52}$, M. Mostafá ${ }^{91}$, A.L. Müller ${ }^{8}$, M.A. Muller ${ }^{22}$, K. Mulrey ${ }^{15}$, R. Mussa ${ }^{52}$, M. Muzio ${ }^{90}$, W.M. Namasaka ${ }^{38}$, A. Nasr-Esfahani ${ }^{38}$, L. Nellen ${ }^{68}$, M. Niculescu-Oglinzanu ${ }^{73}$, M. Niechciol ${ }^{44}$, D. Nitz ${ }^{89}$, D. Nosek ${ }^{31}$, V. Novotny ${ }^{31}$, L. Nožka ${ }^{33}$, A Nucita ${ }^{56,48}$, L.A. Núñez ${ }^{30}$, M. Palatka ${ }^{32}$, J. Pallotta ${ }^{2}$, P. Papenbreer ${ }^{38}$, G. Parente ${ }^{79}$, A. Parra ${ }^{64}$, J. Pawlowsky ${ }^{38}$, M. Pech ${ }^{32}$, F. Pedreira ${ }^{79}$, J. Pȩkala ${ }^{70}$, R. Pelayo ${ }^{65}$, J. Peña-Rodriguez ${ }^{30}$, E.E. Pereira Martins ${ }^{39,8}$, J. Perez Armand ${ }^{21}$, C. Pérez Bertolli $^{8,41}$, M. Perlin ${ }^{8,41}$, L. Perrone ${ }^{56,48}$, S. Petrera ${ }^{45,46}$, T. Pierog ${ }^{41}$, M. Pimenta ${ }^{72}$, V. Pirronello ${ }^{58,47}$, M. Platino ${ }^{8}$, B. Pont $^{80}$, M. Pothast ${ }^{81,80}$, P. Privitera ${ }^{92}$, M. Prouza ${ }^{32}$, A. Puyleart ${ }^{89}$, S. Querchfeld ${ }^{38}$, J. Rautenberg ${ }^{38}$, D. Ravignani ${ }^{8}$, M. Reininghaus ${ }^{41,8}$, J. Ridky ${ }^{32}$, F. Riehn ${ }^{72}$, M. Risse ${ }^{44}$, V. Rizi ${ }^{57,46}$, W. Rodrigues de Carvalho ${ }^{21}$, J. Rodriguez Rojo ${ }^{11}$, M.J. Roncoroni ${ }^{8}$, S. Rossoni ${ }^{43}$, M. Roth ${ }^{41}$, E. Roulet ${ }^{1}$, A.C. Rovero ${ }^{5}$, P. Ruehl ${ }^{44}$, A. Saftoiu ${ }^{73}$, F. Salamida ${ }^{57,46}$, H. Salazar ${ }^{64}$, G. Salina ${ }^{51}$, J.D. Sanabria Gomez ${ }^{30}$, F. Sánchez ${ }^{8}$, E.M. Santos ${ }^{21}$, E. Santos ${ }^{32}$, F. Sarazin ${ }^{86}$, R. Sarmento ${ }^{72}$, C. Sarmiento-Cano ${ }^{8}$, R. Sato ${ }^{11}$, 
P. Savina ${ }^{56,48,34,94}$, C.M. Schäfer ${ }^{41}$, V. Scherini ${ }^{56,48}$, H. Schieler ${ }^{41}$, M. Schimassek ${ }^{39,8}$, M. Schimp ${ }^{38}$, F. Schlüter ${ }^{41,8}$, D. Schmidt ${ }^{39}$, O. Scholten ${ }^{84,15}$, P. Schovánek ${ }^{32}$, F.G. Schröder ${ }^{93,41}$, S. Schröder ${ }^{38}$, J. Schulte ${ }^{42}$, S.J. Sciutto ${ }^{4}$, M. Scornavacche ${ }^{8,41}$, A. Segreto ${ }^{53,47}$, S. Sehgal ${ }^{38}$, R.C. Shellard ${ }^{16}$, G. Sigl ${ }^{43}$, G. Silli ${ }^{8,41}$, O. Sima ${ }^{73, f}$, R. Šmída ${ }^{92}$, P. Sommers ${ }^{91}$, J.F. Soriano ${ }^{87}$, J. Souchard ${ }^{36}$, R. Squartini ${ }^{10}$, M. Stadelmaier ${ }^{41,8}$, D. Stanca ${ }^{73}$, S. Stanič ${ }^{76}$, J. Stasielak ${ }^{70}$, P. Stassi ${ }^{36}$, A. Streich ${ }^{39,8}$, M. Suárez-Durán ${ }^{14}$, T. Sudholz ${ }^{13}$, T. Suomijärvi ${ }^{37}$, A.D. Supanitsky ${ }^{8}$, Z. Szadkowski ${ }^{71}$, A. Tapia ${ }^{29}$, C. Taricco ${ }^{63,52}$, C. Timmermans ${ }^{81,80}$, O. Tkachenko ${ }^{41}$, P. Tobiska ${ }^{32}$, C.J. Todero Peixoto ${ }^{19}$, B. Tomé ${ }^{72}$, Z. Torrès ${ }^{36}$, A. Travaini ${ }^{10}$, P. Travnicek $^{32}$, C. Trimarelli ${ }^{57,46}$, M. Tueros ${ }^{4}$, R. Ulrich ${ }^{41}$, M. Unger ${ }^{41}$, L. Vaclavek ${ }^{33}$, M. Vacula ${ }^{33}$, J.F. Valdés Galicia ${ }^{68}$, L. Valore ${ }^{60,50}$, E. Varela ${ }^{64}$, A. Vásquez-Ramírez ${ }^{30}$, D. Veberič ${ }^{41}$, C. Ventura ${ }^{27}$, I.D. Vergara Quispe ${ }^{4}$, V. Verzi ${ }^{51}$, J. Vicha ${ }^{32}$, J. Vink $^{83}$, S. Vorobiov ${ }^{76}$, H. Wahlberg ${ }^{4}$, C. Watanabe ${ }^{26}$, A.A. Watson ${ }^{c}$, M. Weber ${ }^{40}$, A. Weindl ${ }^{41}$, L. Wiencke ${ }^{86}$, H. Wilczyński ${ }^{70}$, M. Wirtz ${ }^{42}$, D. Wittkowski ${ }^{38}$, B. Wundheiler ${ }^{8}$, A. Yushkov $^{32}$, O. Zapparrata ${ }^{14}$, E. Zas ${ }^{79}$, D. Zavrtanik ${ }^{76,77}$, M. Zavrtanik ${ }^{77,76}$, L. Zehrer ${ }^{76}$

${ }^{1}$ Centro Atómico Bariloche and Instituto Balseiro (CNEA-UNCuyo-CONICET), San Carlos de Bariloche, Argentina

${ }^{2}$ Centro de Investigaciones en Láseres y Aplicaciones, CITEDEF and CONICET, Villa Martelli, Argentina

${ }^{3}$ Departamento de Física and Departamento de Ciencias de la Atmósfera y los Océanos, FCEyN, Universidad de Buenos Aires and CONICET, Buenos Aires, Argentina

${ }^{4}$ IFLP, Universidad Nacional de La Plata and CONICET, La Plata, Argentina

${ }^{5}$ Instituto de Astronomía y Física del Espacio (IAFE, CONICET-UBA), Buenos Aires, Argentina

${ }^{6}$ Instituto de Física de Rosario (IFIR) - CONICET/U.N.R. and Facultad de Ciencias Bioquímicas y Farmacéuticas U.N.R., Rosario, Argentina

${ }^{7}$ Instituto de Tecnologías en Detección y Astropartículas (CNEA, CONICET, UNSAM), and Universidad Tecnológica Nacional - Facultad Regional Mendoza (CONICET/CNEA), Mendoza, Argentina

${ }^{8}$ Instituto de Tecnologías en Detección y Astropartículas (CNEA, CONICET, UNSAM), Buenos Aires, Argentina

${ }^{9}$ International Center of Advanced Studies and Instituto de Ciencias Físicas, ECyT-UNSAM and CONICET, Campus Miguelete - San Martín, Buenos Aires, Argentina

${ }^{10}$ Observatorio Pierre Auger, Malargüe, Argentina

${ }^{11}$ Observatorio Pierre Auger and Comisión Nacional de Energía Atómica, Malargüe, Argentina

12 Universidad Tecnológica Nacional - Facultad Regional Buenos Aires, Buenos Aires, Argentina

${ }^{13}$ University of Adelaide, Adelaide, S.A., Australia

14 Université Libre de Bruxelles (ULB), Brussels, Belgium

15 Vrije Universiteit Brussels, Brussels, Belgium

${ }^{16}$ Centro Brasileiro de Pesquisas Fisicas, Rio de Janeiro, RJ, Brazil

${ }^{17}$ Centro Federal de Educação Tecnológica Celso Suckow da Fonseca, Nova Friburgo, Brazil

${ }^{18}$ Instituto Federal de Educação, Ciência e Tecnologia do Rio de Janeiro (IFRJ), Brazil

${ }^{19}$ Universidade de São Paulo, Escola de Engenharia de Lorena, Lorena, SP, Brazil

${ }^{20}$ Universidade de São Paulo, Instituto de Física de São Carlos, São Carlos, SP, Brazil

${ }^{21}$ Universidade de São Paulo, Instituto de Física, São Paulo, SP, Brazil

${ }^{22}$ Universidade Estadual de Campinas, IFGW, Campinas, SP, Brazil

${ }^{23}$ Universidade Estadual de Feira de Santana, Feira de Santana, Brazil

${ }^{24}$ Universidade Federal do ABC, Santo André, SP, Brazil

${ }^{25}$ Universidade Federal do Paraná, Setor Palotina, Palotina, Brazil

${ }^{26}$ Universidade Federal do Rio de Janeiro, Instituto de Física, Rio de Janeiro, RJ, Brazil

${ }^{27}$ Universidade Federal do Rio de Janeiro (UFRJ), Observatório do Valongo, Rio de Janeiro, RJ, Brazil

${ }^{28}$ Universidade Federal Fluminense, EEIMVR, Volta Redonda, RJ, Brazil

${ }^{29}$ Universidad de Medellín, Medellín, Colombia

${ }^{30}$ Universidad Industrial de Santander, Bucaramanga, Colombia

${ }^{31}$ Charles University, Faculty of Mathematics and Physics, Institute of Particle and Nuclear Physics, Prague, Czech Republic

32 Institute of Physics of the Czech Academy of Sciences, Prague, Czech Republic 
33 Palacky University, RCPTM, Olomouc, Czech Republic

34 CNRS/IN2P3, IJCLab, Université Paris-Saclay, Orsay, France

${ }^{35}$ Laboratoire de Physique Nucléaire et de Hautes Energies (LPNHE), Sorbonne Université, Université de Paris, CNRSIN2P3, Paris, France

${ }^{36}$ Univ. Grenoble Alpes, CNRS, Grenoble Institute of Engineering Univ. Grenoble Alpes, LPSC-IN2P3, 38000 Grenoble, France

${ }^{37}$ Université Paris-Saclay, CNRS/IN2P3, IJCLab, Orsay, France

38 Bergische Universität Wuppertal, Department of Physics, Wuppertal, Germany

${ }^{39}$ Karlsruhe Institute of Technology (KIT), Institute for Experimental Particle Physics, Karlsruhe, Germany

${ }^{40}$ Karlsruhe Institute of Technology (KIT), Institut für Prozessdatenverarbeitung und Elektronik, Karlsruhe, Germany

${ }^{41}$ Karlsruhe Institute of Technology (KIT), Institute for Astroparticle Physics, Karlsruhe, Germany

${ }^{42}$ RWTH Aachen University, III. Physikalisches Institut A, Aachen, Germany

${ }^{43}$ Universität Hamburg, II. Institut für Theoretische Physik, Hamburg, Germany

${ }^{44}$ Universität Siegen, Department Physik - Experimentelle Teilchenphysik, Siegen, Germany

${ }^{45}$ Gran Sasso Science Institute, L'Aquila, Italy

46 INFN Laboratori Nazionali del Gran Sasso, Assergi (L'Aquila), Italy

${ }^{47}$ INFN, Sezione di Catania, Catania, Italy

${ }^{48}$ INFN, Sezione di Lecce, Lecce, Italy

${ }^{49}$ INFN, Sezione di Milano, Milano, Italy

${ }^{50}$ INFN, Sezione di Napoli, Napoli, Italy

${ }^{51}$ INFN, Sezione di Roma "Tor Vergata", Roma, Italy

52 INFN, Sezione di Torino, Torino, Italy

53 Istituto di Astrofisica Spaziale e Fisica Cosmica di Palermo (INAF), Palermo, Italy

54 Osservatorio Astrofisico di Torino (INAF), Torino, Italy

55 Politecnico di Milano, Dipartimento di Scienze e Tecnologie Aerospaziali, Milano, Italy

${ }^{56}$ Università del Salento, Dipartimento di Matematica e Fisica "E. De Giorgi”, Lecce, Italy

57 Università dell'Aquila, Dipartimento di Scienze Fisiche e Chimiche, L'Aquila, Italy

58 Università di Catania, Dipartimento di Fisica e Astronomia, Catania, Italy

${ }^{59}$ Università di Milano, Dipartimento di Fisica, Milano, Italy

${ }^{60}$ Università di Napoli "Federico II", Dipartimento di Fisica "Ettore Pancini”, Napoli, Italy

${ }^{61}$ Università di Palermo, Dipartimento di Fisica e Chimica "E. Segrè”, Palermo, Italy

62 Università di Roma "Tor Vergata", Dipartimento di Fisica, Roma, Italy

${ }^{63}$ Università Torino, Dipartimento di Fisica, Torino, Italy

${ }^{64}$ Benemérita Universidad Autónoma de Puebla, Puebla, México

${ }^{65}$ Unidad Profesional Interdisciplinaria en Ingeniería y Tecnologías Avanzadas del Instituto Politécnico Nacional (UPIITA-IPN), México, D.F., México

66 Universidad Autónoma de Chiapas, Tuxtla Gutiérrez, Chiapas, México

${ }^{67}$ Universidad Michoacana de San Nicolás de Hidalgo, Morelia, Michoacán, México

${ }^{68}$ Universidad Nacional Autónoma de México, México, D.F., México

${ }^{69}$ Universidad Nacional de San Agustin de Arequipa, Facultad de Ciencias Naturales y Formales, Arequipa, Peru

${ }^{70}$ Institute of Nuclear Physics PAN, Krakow, Poland

${ }^{71}$ University of Łódź, Faculty of High-Energy Astrophysics, Łódź, Poland

${ }^{72}$ Laboratório de Instrumentação e Física Experimental de Partículas - LIP and Instituto Superior Técnico - IST, Universidade de Lisboa - UL, Lisboa, Portugal

73 "Horia Hulubei” National Institute for Physics and Nuclear Engineering, Bucharest-Magurele, Romania

${ }^{74}$ Institute of Space Science, Bucharest-Magurele, Romania

75 University Politehnica of Bucharest, Bucharest, Romania

76 Center for Astrophysics and Cosmology (CAC), University of Nova Gorica, Nova Gorica, Slovenia

${ }^{77}$ Experimental Particle Physics Department, J. Stefan Institute, Ljubljana, Slovenia

78 Universidad de Granada and C.A.F.P.E., Granada, Spain

${ }^{79}$ Instituto Galego de Física de Altas Enerxías (IGFAE), Universidade de Santiago de Compostela, Santiago de Compostela, Spain 
${ }^{80}$ IMAPP, Radboud University Nijmegen, Nijmegen, The Netherlands

${ }^{81}$ Nationaal Instituut voor Kernfysica en Hoge Energie Fysica (NIKHEF), Science Park, Amsterdam, The Netherlands

${ }^{82}$ Stichting Astronomisch Onderzoek in Nederland (ASTRON), Dwingeloo, The Netherlands

${ }^{83}$ Universiteit van Amsterdam, Faculty of Science, Amsterdam, The Netherlands

${ }^{84}$ University of Groningen, Kapteyn Astronomical Institute, Groningen, The Netherlands

85 Case Western Reserve University, Cleveland, OH, USA

86 Colorado School of Mines, Golden, CO, USA

${ }^{87}$ Department of Physics and Astronomy, Lehman College, City University of New York, Bronx, NY, USA

${ }^{88}$ Louisiana State University, Baton Rouge, LA, USA

${ }^{89}$ Michigan Technological University, Houghton, MI, USA

${ }^{90}$ New York University, New York, NY, USA

${ }^{91}$ Pennsylvania State University, University Park, PA, USA

92 University of Chicago, Enrico Fermi Institute, Chicago, IL, USA

93 University of Delaware, Department of Physics and Astronomy, Bartol Research Institute, Newark, DE, USA

${ }^{94}$ University of Wisconsin-Madison, Department of Physics and WIPAC, Madison, WI, USA

${ }^{a}$ Fermi National Accelerator Laboratory, Fermilab, Batavia, IL, USA

${ }^{b}$ Max-Planck-Institut für Radioastronomie, Bonn, Germany

${ }^{c}$ School of Physics and Astronomy, University of Leeds, Leeds, United Kingdom

${ }^{d}$ Colorado State University, Fort Collins, CO, USA

$e^{e}$ now at Hakubi Center for Advanced Research and Graduate School of Science, Kyoto University, Kyoto, Japan

$f$ also at University of Bucharest, Physics Department, Bucharest, Romania 